\title{
Innovative Tools and Processes for Mobile Communications Research and Education
}

\author{
http://dx.doi.org/10.3991/ijoe.v12i09.6098 \\ F.J. Rivas-Tocado ${ }^{1}$, A. Diaz-Zayas ${ }^{2}$ and P.Merino ${ }^{2}$ \\ ${ }^{1}$ Keysight Technologies, Malaga, Spain \\ 2 University of Malaga, Andalucía Tech, Málaga, Spain
}

\begin{abstract}
Coming generations of engineering graduates and researchers will be expected to develop an integral set of skills to adapt themselves to the mobile communications industry and market expectations. To meet these demands universities will need to collaborate and innovate in their education styles, research tools and processes. The evolution of remote experimentation and the availability of state-ofthe-art mobile communications test systems bring new opportunities to this field, such as experimentation with Gigabit class LTE-A. In this paper we discuss how this equipment could be adopted for educational purposes and how it can be included in federated research environments.
\end{abstract}

Index Terms-UXM, mobile communications, test systems, remote labs, federation.

\section{INTRODUCTION}

The fast evolution and adoption of LTE and the forthcoming specification of $5 \mathrm{G}$ are evident signs of the increasing speed of change in mobile technologies. As a clear example Keysight Technologies and Qualcomm have jointly demonstrated 1 Gbps connectivity over LTEA during the Mobile World Congress 2016 in Barcelona [1]. Each new technology development brings with it fresh challenges that the new generations of engineers will have to face, e.g. with mobile devices- applications and protocols having to deal with unprecedented data rates that may require significant re-architecture work. In the authors' experience, current educational processes and resources are sometimes lacking in very important market-ready competencies. In this dynamic context it is critical that new engineers receive the most up-to-date education and researchers have access to cutting edge instruments to obtain relevant results. This is not only a challenging scenario where universities will need to differentiate themselves to attract students, but also an opportunity for them to collaborate and share resources.

In [2] Castro et al include an insightful overview of the evolution of trends and advances in engineering education over the last few decades. Interestingly, they highlight that universities may have been reluctant to address the specific needs of industry and labor market in the past. However, a change of paradigm is being addressed by the use of internet and mobile technologies to provide ubiquitous and life-long learning opportunities in a student-centered approach.

The need for institutional collaboration to achieve stateof-the-art market-oriented education is not only specific to the telecommunications domain, but it is a constant in many other sectors. An interesting example of collaboration in the field of industrial automation is the TATU project [3]. TATU (Training in Automation Technology for Ukraine) is an EU TEMPUS IV project part of the Higher Education and Society Action 2013 - 2016. TATU's aim is to enhance the employability of university graduates from 5 Ukrainian universities and Life Long Learning (LLL) in the field of industrial automation through the introduction of European standards of education through practical examples.

In the scope of wireless and radio communications [4] is a meaningful example of antennae experimentation through collaboration in the context of an interinstitutional online master's degree program [5]. This program has been funded by the RIPLECS European project with a distinct focus on high market orientation, in four different countries.

The European Commission has promoted e-Science and related ICT research for years [6]. Further examples are the Communication from the EU Commissions on ICT infrastructures for e-Science, and Digital Agenda for Europe.

Remote experimentation is receiving increasing attention in engineering because it increases lab availability, maximizing the use of the equipment and enabling distance learning [1] [2] [4] [5] [7] [8]. There are different types of remote labs, often based on virtual web-based, simulated experiments. The use of computer simulations have been found to be useful to save costs, but there are arguments about their effectiveness compared with access to real equipment [9], thus they should be combined with real labs for increased student benefit instead of being a complete replacement [5].

A more realistic approach has been followed by remote experimentation labs that have actual traditional instruments including signal generators and signal analyzers to generate and measure RF signals [1][10]. In [7] the authors highlight the benefits of internet accessible laboratories to achieve an efficient reuse of radio communication equipment. They have also conducted an assessment of the positive effect of remote labs in learning and the benefits for students in terms of flexibility and higher availability.

A Microwave Amplifier Laboratory at the HSE Moscow Institute of Electronics and Mechanics [11] introduces students to the use of a RF signal generator, a signal analyzer and a hi-end oscilloscope from Keysight Technologies. However to the best of our knowledge no remote education lab has provided access to mobile network emulators to gain insight into the actual operation of mobile phones in a controlled environment.

The rest of the paper is organized as follows. Section II introduces the concept of mobile network emulation. Section III describes the key features provided by the UXM wireless test set, illustrating how they could fit into 
an educational and research context. Section IV shows how the University of Malaga has integrated mobile communications test systems into the PerformNetworks testbed using Future Internet Research \& Experimentation (FIRE) technologies.

\section{MOBILE NeTwORK EMUlation}

Although not widely present in an academic context outside of the industry, mobile phones pass through a demanding test process before receiving approval to be used in mobile networks. For that purpose, the standardization organizations like 3 GPP $\left(3^{\text {rd }}\right.$ Generation Partnership Project) define extensive conformance specifications. Advanced test platforms like the UXM [12] Wireless Test Set from Keysight Technologies, shown in Error! Reference source not found., implement these tests, behaving as real base stations from the standpoint of the mobile phones being tested. In addition, mobile device manufacturers and other UXM users also benefit from a constantly increasing variety of features intended to provide a powerful and flexible R\&D test environment.

Despite wireless test sets not having been widely used in the past in universities, their increasing functionality and modularity are making them more cost effective equipment. In other words, the availability of embedded digital radio channel emulation reduces the complexity of the RF test setups and improves the accuracy with a lower cost compared to traditional external RF emulators. In addition, as these devices are designed to operate on a $24 / 7$ basis, the required investment can also be greatly reduced by sharing them both for research and education purposes even during traditionally off hours.

Unlike legacy RF instruments, the impact of using UXMs could span a wider number of areas, ranging from signal processing to radio channel emulation, also including protocol and mobility evaluation as well as end-to-end application traffic analysis [13]. This would also extend the reuse potential to multiple subjects and labs, reducing the ownership cost accordingly. Another direct benefit would be the improvement in the learning curve of successive laboratories based on the same underlying equipment.

In the context of the European FP7 project Fed4Fire, the PerformNetworks facility has, for remote research purposes, developed a test environment based on the Keysight T2010 legacy base station emulator [14]. Leveraging the experience gained using base station emulators we propose a wider adoption of this type of equipment in a university context as a complement to traditional instrumentation and software simulation tools [15].

\section{APPLICABILITY OF UXM TO EXPERIMENTAL LABORATORY ACTIVITIES}

The UXM is an extremely versatile instrument. It is able to emulate multiple base stations with different radio access technologies, including LTE/LTE-A, WCDMA/HSPA+, GSM/GPRS/EGPRS and TDSCDMA/HSPA. Additionally it can also operate simultaneously as a radio channel emulator, noise and arbitrary waveform generator to generate impairments and as a signal analyzer.

One of the key UXM advantages is the ease of use of the E7530A and E7630A LTE/LTE-A Test and Lab Applications [16] that run inside the instrument itself. When

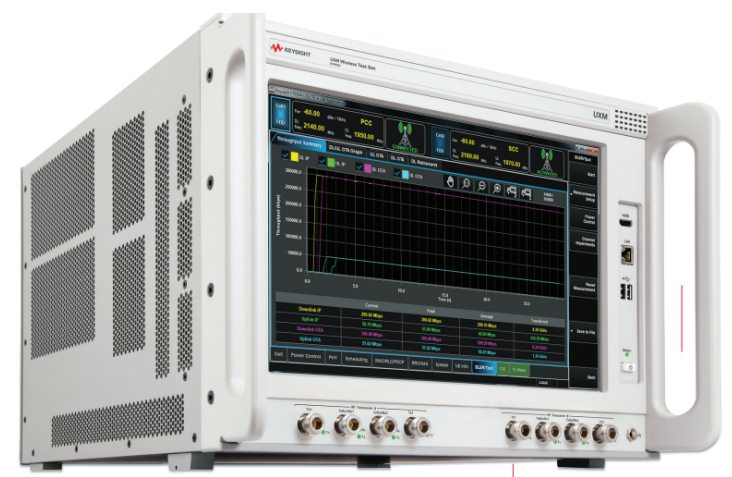

Figure 1. UXM Wireless Test Set

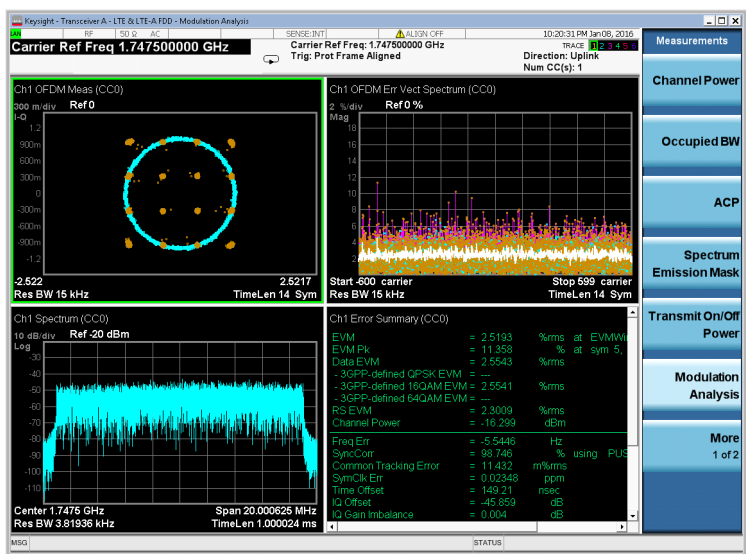

Figure 2. XApps measurement tools

being used locally, the windows based GUI can also be accessed through its large touch screen. When used remotely, it can be operated via Standard Commands for Programmable Instruments (SCPI) and also through a Remote Desktop access.

Next we briefly introduce some of the key features of UXM that show a high potential for research and education in mobile communications. Each feature could be the basis for different experimental laboratories.

\section{A. Transmitter RF and Baseband Signal Analysis}

Among its many capabilities UXM provides native support for Keysight's XApps measurement tools that are the de-facto standard for signal analysis. A myriad of key concepts related to the phone transmitter could be introduced to students, such as Error Vector Magnitude (EVM) measurements, spectrum analysis, equalizer flatness, IQ constellations, measurement of dynamic power control and many more. Figure 2 shows a modulation analysis measurement of a $20 \mathrm{MHz} 16 \mathrm{QAM}$ uplink signal.

\section{B. Channel Emulation \& Receiver performance Analysis}

The receivers of mobile devices are typically evaluated in terms of the probability of receiving data in the presence of radio impairments with an error ratio below defined thresholds.

To replicate the effect of radio propagation conditions, the UXM features an embedded digital channel emulator that provides greater simplicity and improves accuracy by avoiding the uncertainty contributions typically encountered in external RF interconnections.

In addition to the emulation of fading scenarios, other impairments can be generated including $\mathrm{CW}$ (Continuous 
Waveform), AWGN (Additive White Gaussian Noise) and arbitrary waveforms.

Figure 3 shows a logical diagram of the channel emulation and impairment addition for a configuration including 2 Component Carriers (CC) with $4 \times 2$ Multiple Input Multiple Output (MIMO) antenna configuration, representing 4 transmitting antennas in the base station and 2 receiving antennas in the mobile phone. Other supported scenarios in a single UXM include up to $4 \mathrm{CC}$ with $2 \times 2$ MIMO or $2 \mathrm{CC}$ with $4 \times 4$ MIMO.

\section{Mobility Procedures}

As shown in Figure 4, multiple cells can be generated in a single UXM, including multiple Radio Access Technologies (RAT), providing an intuitive and easy to use touch interface.

Understanding the internals of mobile network signaling protocol exchanges may be a complex task if not harnessed to real examples.

In addition to introducing basic concepts such as carrier frequency, frequency bands and channel bandwidths, it is straightforward to perform and monitor many mobility procedures such as network attach, detach or cell reselections. Another notable example could be the evaluation of different types of handovers (intra-band, inter-band, interRAT) upon realistic controlled radio impairments with fading and noise.

\section{End to End IP performance analysis}

Smartphones are no longer expected to only be used for voice calls. Instead, IP based multimedia connections are now prevalent. Another key characteristic of the current use cases is the wide variety of new different user profiles and traffic patterns.

In this scenario it is important to ensure that mobile devices can transmit data up to their maximum published capabilities. UXM features are evolving continuously to help researchers and engineers understand the interactions between applications, operating systems and protocol stacks. As an example, throughputs as high as those of state-of-the-art LTE-A Gigabit class can be tested [1].

It is possible to gain insight into E2E traffic dynamics using real-time monitoring graphs. They illustrate the evolution of the IP throughput and the related MAC level throughput statistics. Figure 5 contains throughput graphs for a 3CC setup with 2x2 MIMO and 256QAM modulation in the DL. The 6 resulting MIMO flows can transport close to $600 \mathrm{Mbps}$.

This type of representation is particularly useful for detecting traffic artifacts that could be related to complex cross layer interactions affecting application level communication performance.

\section{E. Protocol logging and Analysis}

For an in-depth traffic analysis, UXM can deploy powerful protocol and logging analysis software [18], the E7515A-L01. This software allows UXM users to control the generation and filtering of logs from a wireshark integrated interface.

Complex traffic patterns can be analyzed in combination with UXM's ability to emulate realistic radio propagation conditions.

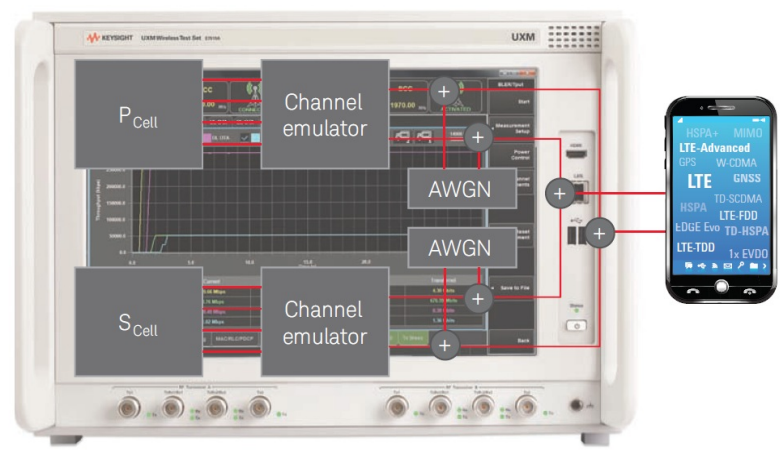

Figure 3. Internal digital radio channel emulation

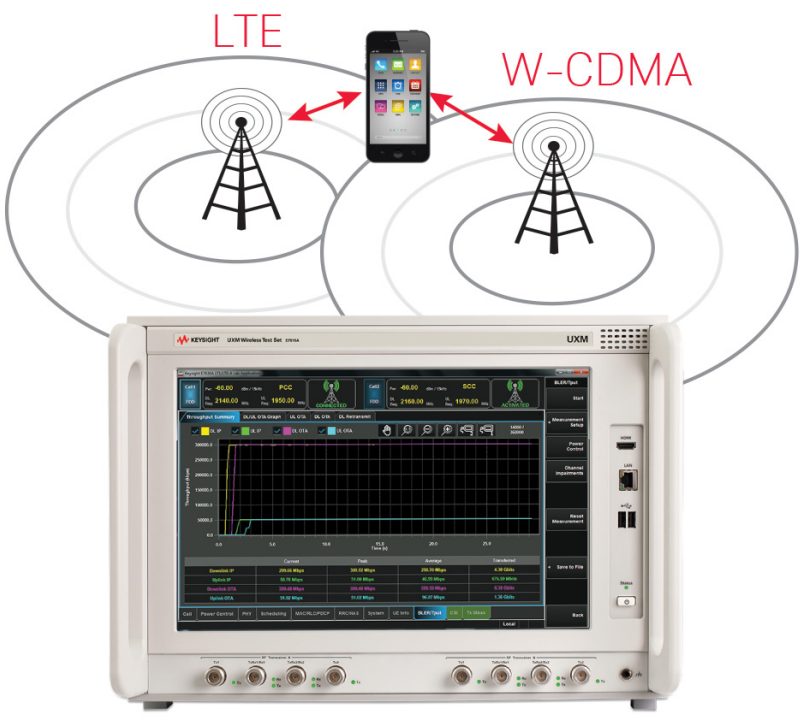

Figure 4. UXM driven mobility procedures

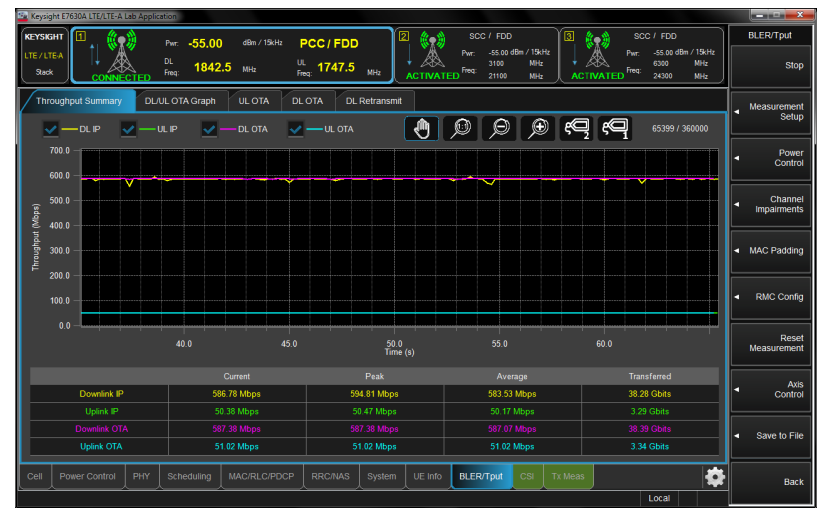

Figure 5. E2E IP and MAC level throughput graphs

It is not only possible to analyze upper layer signaling, including Radio Resource Control (RRC) and Non Access Stratum (NAS), but also the underlying control information and MAC PDUs. An even more sophisticated analysis can be obtained from the correlation of the user plane performance data with the control plane information.

Figure 6 provides an example including RRC messages, like those for system information broadcasting and RRC connection request, as well as detailed information about control channels operation. 
Of particular interest is the use of the integrated flow analysis functions to study the dynamic evolution of TCP flows from the information provided by the MAC-LTE layer as shown in Figure 8.

\section{F. VoLTE and Multimedia testing}

UXM provides multiple radio access features required by the VoLTE profile [17], including SPS (SemiPersistent Scheduling), multiple DRBs (Dedicated Radio Bearers), DRX (Discontinuous Reception) and many more.

As an all IP network, LTE relies on IMS (IP Multimedia Subsystem) to register IMS capable UEs and to establish multimedia sessions.

UXM also integrates an IMS-SIP server [13] that can be combined with an IMS-SIP software client to test VoLTE and other multimedia functional scenarios. Figure 7 illustrates the different tools for audio testing over IP.

\section{G. Heterogeneous Networking}

Heterogeneous networking has been a research area with tremendous activity for some time. These scenarios typically combine multiple cells with different powers, where they are required to coordinate, over time, their transmission patterns to preserve quality, particularly in the cell borders, using enhanced Inter-Cell Interference Coordination (eICIC).

For that purpose, mobile devices can be instructed to measure signal quality and power at different sets of time intervals. A special low power transmission scheme called ABS (Almost Blank Subframes) has also been added to reduce the interference to neighbor cells both in data reception and channel quality estimation. With a correct alignment of the measurement periods to the coordinated ABS transmission patterns, the two MCS (Modulation and Coding Schemes) that will be required can be identified, both when high interference from neighbor cells is present or when their power is reduced or even fully removed in ABS.

Figure 9 provides an example of a $40 \mathrm{~ms}$ interfering pattern where an aggressor cells interleaves full transmission subframes with ABS subframes. Full transmission causes higher interference from the aggressor, whereas ABS allows the phone to receive the signal from the victim cell with reduced interference.

The 3GPP test specifications define a set of reference patterns and cell configurations, but UXM applications provide a highly flexible interface for exploring the performance with complex cell aggression scenarios. It is worth noting that multiple configurations are possible in a cell for ABS to achieve power reduction, where typically, at least CRS (Cell specific Reference Signals) is present for the convenience of the UEs camped in it.

\section{PerformNetworks Testbed}

The PerformNetworks Testbed is a research facility at the University of Malaga that enables experimentation in mobile communications. Among other features, it provides a real but controlled environment involving test mobile network emulators and instrumented Android mobile phones.

This section illustrates how FIRE technologies have been used to include the PerformNetworks testbed within

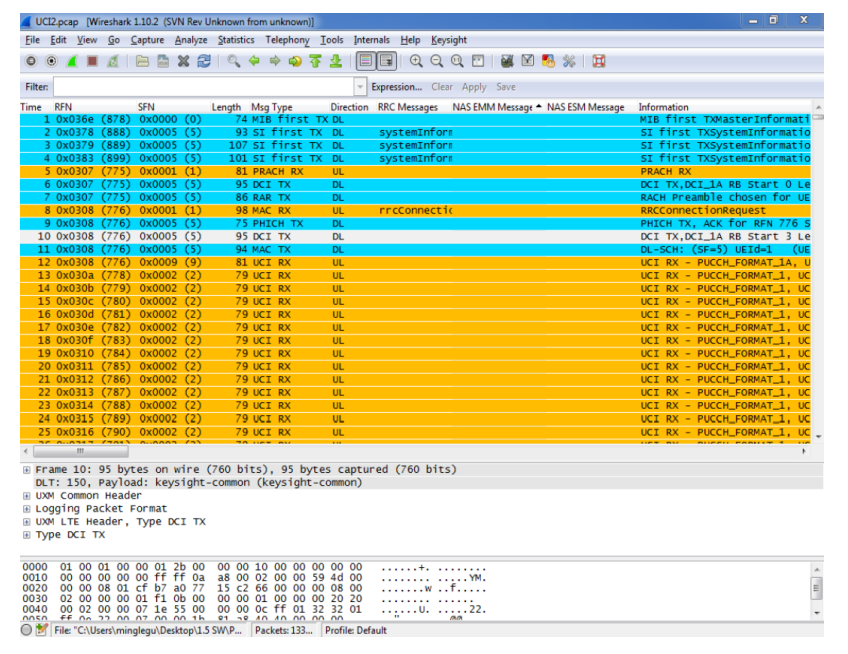

Figure 6. Protocol Logging and Analysis software

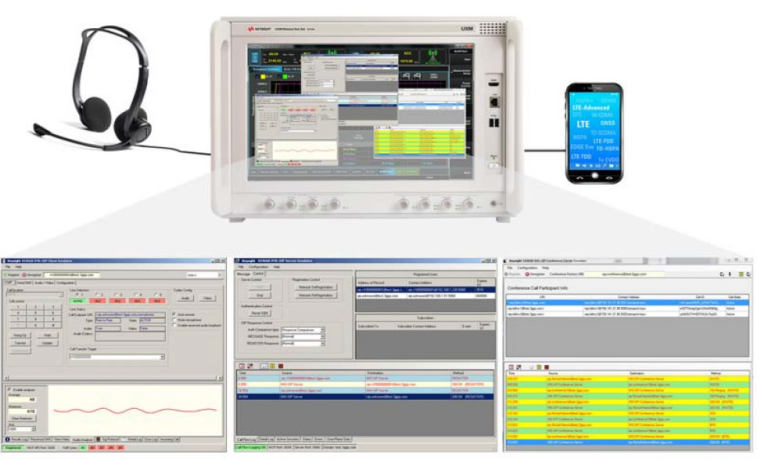

Figure 7. IMS-SIP and VoLTE tools

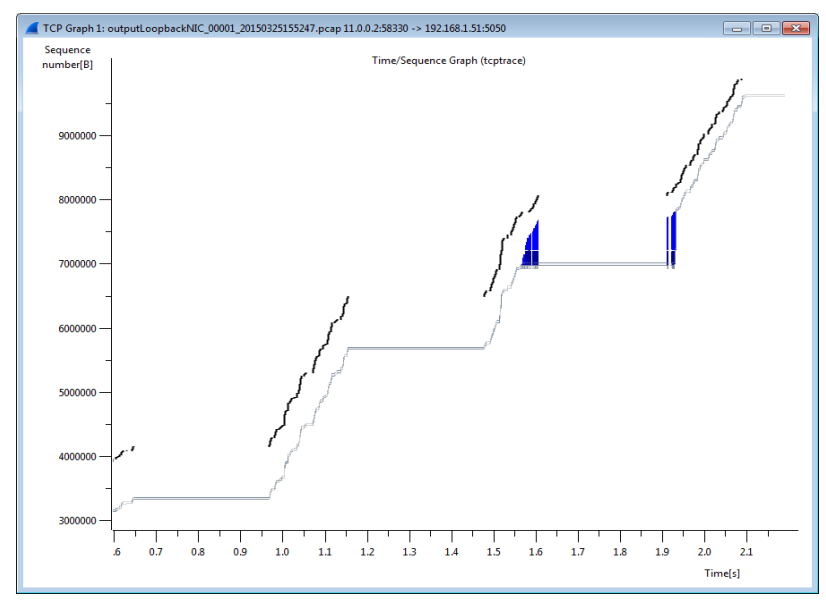

Figure 8. Integration with wireshark tools for data stream analysis

\section{(F)elCIC aggressor test}

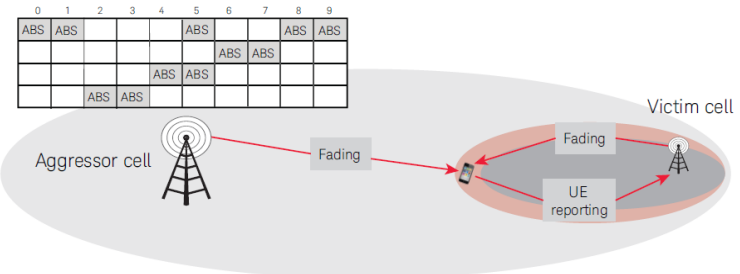

Figure 9. Heterogeneous networking with eICIC

a European research infrastructure. Additionally the workflow of the experiment orchestration within the testbed is depicted. 


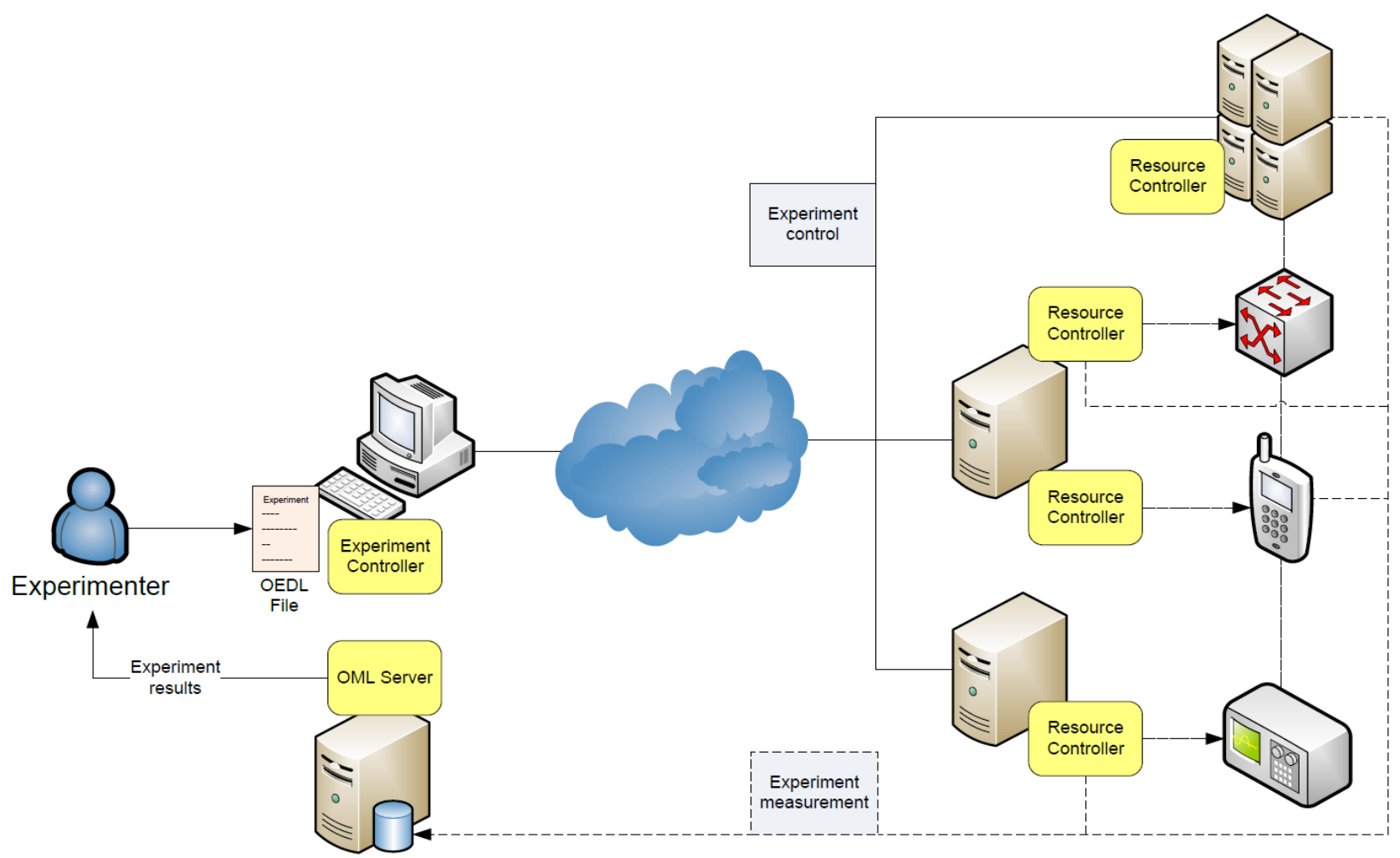

Figure 10. GENI/FIRE testbed Architecture

\section{A. GENI/FIRE Experimentation Technology}

GENI and FIRE are two initiatives for creating a research environment, fostering innovations in networking technologies, the former originating in the United States and the latter in the European Union. Interestingly, both projects are based on the same idea: experimental evaluation is a must in order to produce meaningful research that can be applied to the real world. Thus, the concept of experimental testbeds is central to the implementation of GENI and FIRE. It is worth noting that although GENI and FIRE started as two independent initiatives, Fed4FIRE, a project which is part of FIRE, has adopted GENI technologies, thus making them compatible with each other. The rest of the paper will treat the concepts pertaining to these projects as interchangeable.

Experimentally-driven research demands the ability to perform experiments in an easy and repeatable manner. Testbeds have adopted cOntrol Management Framework ${ }^{1}$ (OMF) and OMF Measurement Library ${ }^{2}$ (OML) as the solutions for providing experiment control and measurement, and experiment management, respectively. The architecture of a generic testbed is shown in Figure 10. An experiment is defined as a file written in the OMF Experiment Description Language (OEDL) ${ }^{3}$. An OEDL file declares the resources that the experiment will use, the events to which it will react, and what actions to perform in response to those events.

The Experiment Controller (EC) interprets OEDL scripts and coordinates the execution of the experiment. Each testbed resource is managed by a Resource Controller (RC), which may be hosted on its own computer or on

\footnotetext{
${ }^{1}$ https://omf.mytestbed.net/

2 https://oml.mytestbed.net/projects/oml/wiki/

${ }^{3}$ https://mytestbed.net/projects/omf6/wiki/OEDLOMF6
}

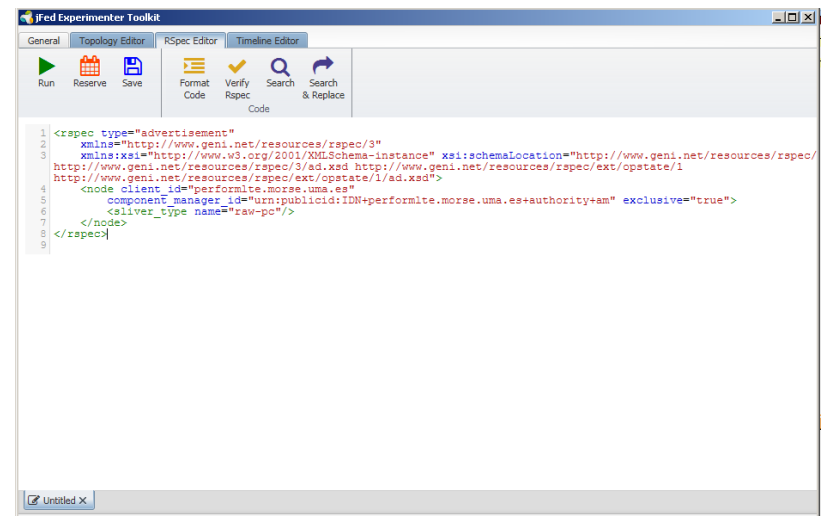

Figure 11. PerformNetwork RSpec

the resource itself. The RCs and the EC exchange control information using the Federated Resource Control Protocol (FRCP), which may be transported over the Extensible Messaging Presence Protocol (XMPP) or the Advanced Message Queuing Protocol (AMQP).

The OML server collects and stores measurements from the experiment. Each instrument, or an RC on behalf of an instrument, can send measurement data using the OML client library. Measurements are collected per experiment and can be queried using standard SQL tools.

Once the experiment script has been defined it is sent to the experiment controller. The experiment controller interacts with the Android resource controller or the Mobile Network Emulator resource controller, to execute the actions defined in the script, in the mobile terminal or test equipment. During the experiment the OML collection server collects all the measurements defined in the script.

The discovery, reservation and provisioning of the resources available in the testbeds are done via Slice-based 
PAPER

InNOVATIVE TOOLS AND PROCESSES FOR MOBILE COMMUNICATIONS RESEARCH AND EDUCATION

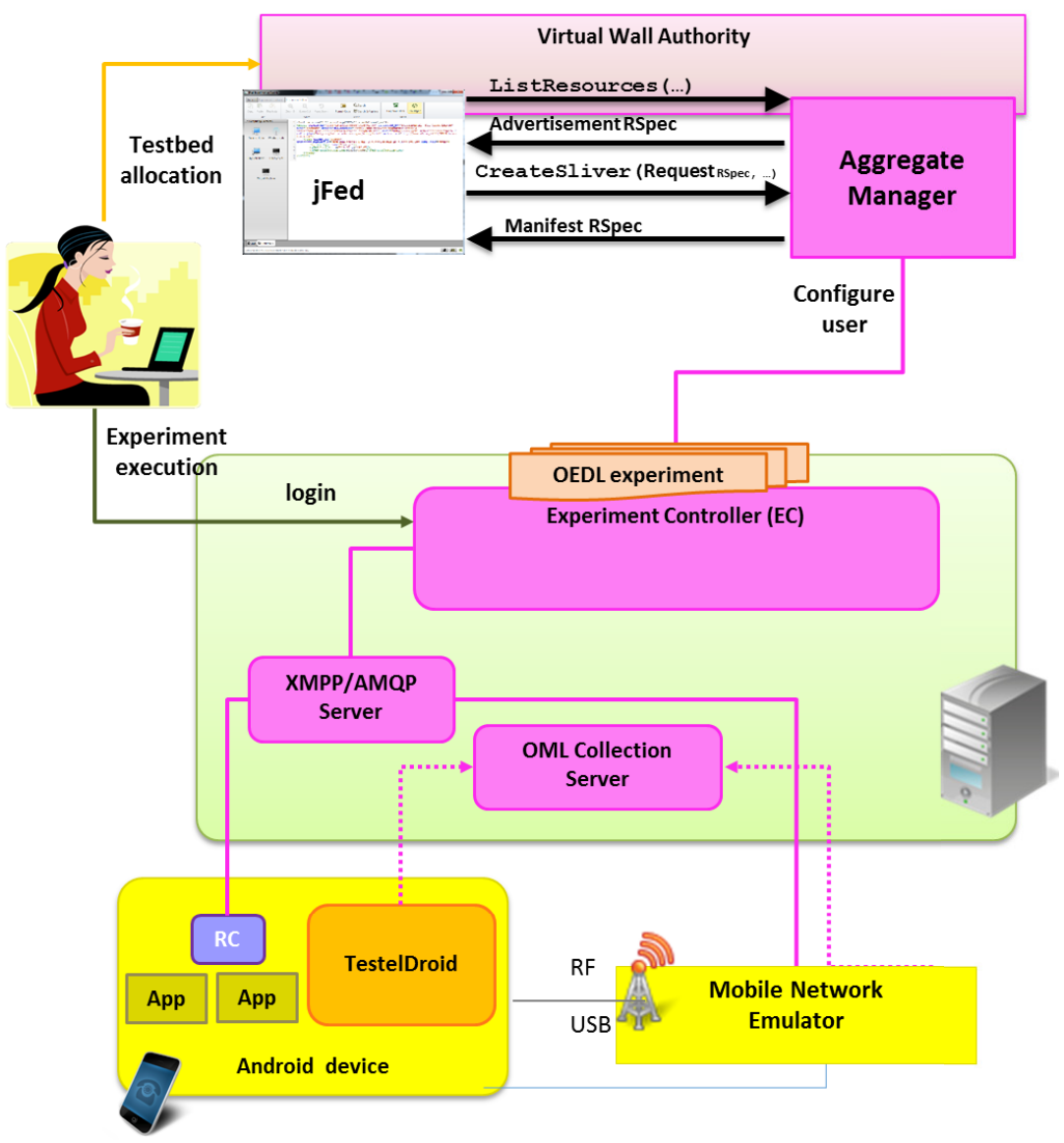

Figure 12. PerformNetwork experiment workflow

Facility Architecture (SFA) [19]. In order to initiate the discovery and reservation processes we need to provide an RSpec (Request Specification) document which contains an XML definition of the resources. The entity defined by the SFA to manage queries about the resources is the Aggregate Manager (AM). The AM is queried by a client tool which implements a compatible AM API such as jFed [15]. The jFed tool allows end-users to provision and manage access via a graphical user interface and a command line interface.

The PerformNetworks experiment workflow is shown in Figure 12 and described in detail in the next section.

\section{B. PerformNetworks Experiment Workflow}

The first step is to obtain an X.509 certificate. The current authority available in the Fed4Fire federation is available at https://authority.ilabt.imind.be. After downloading a valid certificate, the $\mathrm{jFed}$ tool can be used to establish communication with the AM and gain access to the testbed.

The Aggregate Manager (AM) deployed in the testbed is compliant with AM GENI v3 API and its implementation is based on the GCF project [20] which provides a reference implementation of the GENI Aggregate Manager API. The Aggregate Manager of PerformNetworks provides a federated SSH access to the Experiment Controller (EC) of the testbed.

The RSpec definition (see Figure 11) of the testbed provides a monolithic specification of the testbed which enables the allocation of all the resources available. This definition can be used to gain SSH access to the experi-

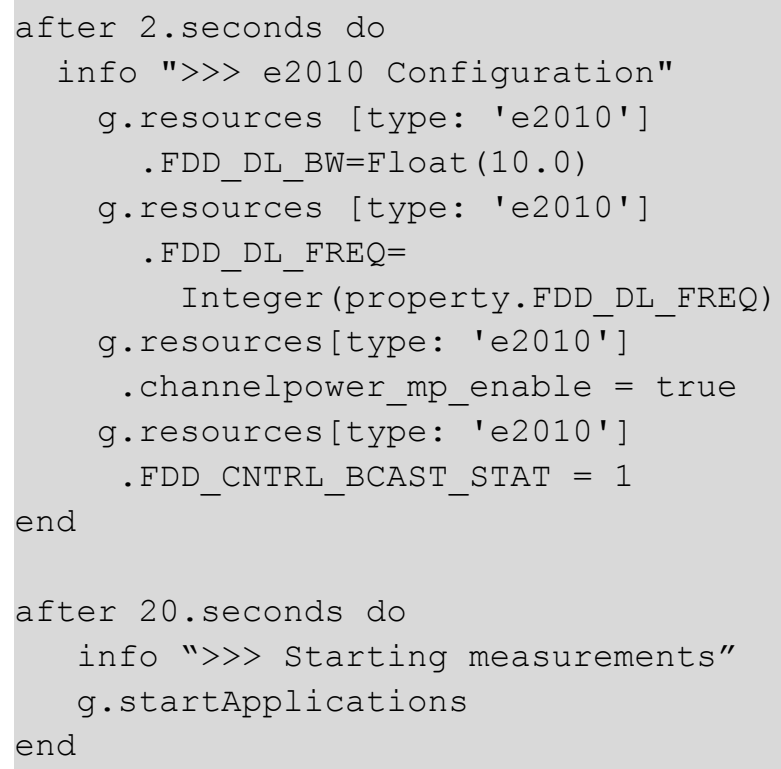

Figure 13. Simple experiment defined using OEDL

ment controller of PerformNetworks using, for example, the $\mathrm{j}$ Fed Experimenter GUI tool.

The experiment controller contains reference experiments described in OEDL (see Figure 13). The experimenters can edit and launch their customized experiments using omf_ec procedures available from the experiment controller $\overline{(1)}$. 


\section{CONCLUSIONS}

To keep pace with mobile technologies, including evolutions as Gigabit class mobile communications, new tools and processes will be required in universities and research centers.

Using innovative equipment such as the UXM wireless test set, both new engineering graduates and researchers will develop and maintain their market oriented competencies. The evolution of education trends and the collaboration initiatives between multiple institutions will also contribute to obtaining the greatest benefit from these new opportunities.

In addition to traditional access schemes such as remote desktop access, we have also illustrated how powerful research federation technologies such as OMF and SFA can be used for experiment handling, orchestrating both mobile phones and mobile network emulators within the PerformNetworks testbed. These technologies share many commonalities with remote educational laboratories, such as the need for resource reservation, access control, and experiment control. In consequence, they could also be considered as alternatives for remote education labs.

\section{REFERENCES}

[1] "Keysight technologies demonstrates gigabit LTE-A download speeds with UXM, Qualcomm Snapdragon X16 LTE modem" Press Release, 2016.

[2] M. Castro, M. Tawfik and E. Tovar, "Digital and global view of engineering education using remote practical competences", IEEE Revista Iberoamericana de Tecnologias del Aprendizaje (RITA), Vol.10, Issue 3, pp 126-133. http://dx.doi.org/10.1109/RITA.2015. 2452651

[3] Workshop: "The TATU lab \& smart education", 13th International Conference on Remote Engineering and Virtual Instrumentation (REV), 2016, pp. 400-402

[4] N. Kafadarova, N. Mileva and S. Stoyanova, "Remote wireless communications lab in real time", IEEE Global Engineering Education Conference (EDUCON), 2013, pp. 6974http://dx.doi.org/10.1109/educon.2013.6530088

[5] M. Tawfik, E. Sancristobal, S. Martin, R. Gil, A. Pesquera et al, "Labor-oriented online master degree program", IEEE Global Engineering Education Conference (EDUCON), 2013, pp. 10981102

[6] M.A. Bochicchio, A. Longo, M. Zappatore and D. Tarantino, "The role of online labs in the european e-sicence infrastructure", 13th International Conference on Remote Engineering and Virtual Instrumentation (REV), 2016, pp. 359 - 365 http://dx.doi.org/10.1109/REV.2016.7444503

[7] A. Gampe, A. Melkonyan, M. Pontual and D. Akiopian , "An assessment of remote laboratory experiments in radio communications", IEEE Transactions on Education, 2014, Volume:57, Issue: 1 , pp. 12- 19

[8] A. Nassar, M. Mohammed, A. Elrashidi and K. Elleithy, "Virtual wireless and mobile communication laboratory", Education, Vol. 2 No. 1, 2012, pp. 19-24. http://dx.doi.org/10.5923/j.edu.20120 201.04

[9] L. D. Feisel and A. J. Rosa, "The role of the laboratory in undergraduate engineering education”, J. Eng. Edu., vol. 94, no. 1, pp. $121-130$

[10] A. Kara, E.U. Aydin, R. Oktem and N. Cagiltay, "A Remote laboratory for training in radio communications: ERRL", IEEE 18th International Symposium on Personal, Indoor and Mobile Radio Communications (PIMRC) 2007, pp 1-5, 3-7 Sept. http://dx.doi.org/10.1109/pimrc.2007.4394833

[11] I. Titov, A. Glotov, Y. Andrey, V. Petrov; "Labicom labs: remote and virtual solid-state laser lab, RF \& microwave amplifier remote and virtual lab", 13th International Conference on Remote Engineering and Virtual Instrumentation (REV), 2016, pp 336-338

[12] Keysight Technologies. E7515A UXM Wireless Test Set [Online]. Available: www.keysight.com/find/uxm, visited on 1 January 2016.

[13] Keysight Technologies,'E6966B IMS-SIP network emulator". Technical Overview. 2015

[14] A. Díaz-Zayas, A.M. Recio-Perez, C.A. García-Perez and P. Merino , PerformLTE: a Testbed for LTE testing in the Future Internet, 13th International Conference on Wired \& Wireless Internet Communications, 2015

[15] F.J. Rivas-Tocado, A. Diaz-Zayas and P. Merino, "Improving mobile communications research and education with UXM wireless test set", 13th International Conference on Remote Engineering and Virtual Instrumentation (REV), 2016, pp. 186189http://dx.doi.org/10.1109/REV.2016.7444462

[16] Keysight Technologies. "E7530A and E7630A LTE/LTE-A Test and Lab Applications". Technical Overview. 2015.

[17] "Official document IR.92 - IMS profile for voice and SMS". GSM Association. 2015.

[18] Keysight Technologies, "E7515A-L01 Protocol logging and analysis software", User's guide. 2014

[19] "Slice-Based Federation Architecture," Version 2.0, GENI Initiative, Tech. Rep., Jul. 2010. [Online]. Available: http://groups.geni.net/geni/wiki/SliceFedArch

[20] B. Vermeulen, W. Van de Meerssche and T. Walcarius, "jFed toolkit, Fed4FIRE, federation"; GENI Engineering Conference (GEC), 2014

[21] GCF project. [Online]. Available: https://github.com/GENINSF/geni-t

\section{AUTHORS}

F. J. Rivas-Tocado is with Keysight Technologies, Malaga, Spain and formerly with AT4 wireless since 2004. He has a Ph.D from the University of Malaga and his research interests include mobile communications protocols and end to end quality of service of IP multimedia traffic (e-mail: javier_rivas@keysight.com).

A. Diaz-Zayas is a post-doctoral researcher with the Department of Languages and Computer Science, Malaga University, Malaga, Spain. Her research interests include mobile communication protocols and experimental facilities (e-mail: almudiaz@lcc.uma.es).

P. Merino Merino is an associate professor at University of Malaga. His research interests are foundations, tools, and applications of formal methods for critical systems, particularly communications software, and development techniques for Internet services on mobile networks. Responsible of several national research projects on mobile phone technologies and embedded systems, he is also the leader of several projects oriented to technology transfer in the field of mobile communications (e-mail: pedro@1cc.uma.es).

This work has been funded by the Government of Andalusia under grant P11-TIC-7659, by the Spanish Ministry of Economy and Competitiveness under project TIN2012-35669 and European Regional Development Fund (EDRF) from the European Commission.

This work was carried out with the support of the Fed4FIRE-project (Federation for FIRE), an Integrated project receiving funding from the European Union's Seventh Framework Programme for research, technological development and demonstration under grant agreement no 318389.

Submitted 03 August 2016. Published as resubmitted by the authors 14 September 2016. 